\title{
The Existence of Solutions to the Nonhomogeneous $A$-Harmonic Equations with Variable Exponent
}

\author{
Haiyu Wen \\ Department of Mathematics, Harbin Institute of Technology, Harbin 150001, China \\ Correspondence should be addressed to Haiyu Wen; wenhy@hit.edu.cn
}

Received 24 October 2013; Revised 16 January 2014; Accepted 17 January 2014; Published 24 February 2014

Academic Editor: Shusen Ding

Copyright (C) 2014 Haiyu Wen. This is an open access article distributed under the Creative Commons Attribution License, which permits unrestricted use, distribution, and reproduction in any medium, provided the original work is properly cited.

We first discuss the existence and uniqueness of weak solution for the obstacle problem of the nonhomogeneous $A$-harmonic equation with variable exponent, and then we obtain the existence of the solutions of the equation $d^{\star} A(x, d \omega)=B(x, d \omega)$ in the weighted variable exponent Sobolev space $W_{d}^{p(x)}\left(\Omega, \Lambda^{l}, \mu\right)$.

\section{Introduction}

In [1-5], the nonhomogeneous $A$-harmonic equation $d^{\star} A(x, d \omega)=B(x, d \omega)$ for differential forms has received much investigation. In [6], the obstacle problem of the $A$ harmonic equation for differential forms has been discussed. However, most of these results are developed in the $L^{p}\left(\Omega, \Lambda^{l}\right)$ space or $W^{1, p}\left(\Omega, \Lambda^{l}\right)$ space. Meanwhile, in the past few years the subject of variable exponent space has undergone a vast development; see [7-11]. For example, [8-10] discuss the weighted $L^{p(x)}$ and $W^{k, p(x)}$ spaces and the weak solution for obstacle problem with variable growth has been studied in $[10,11]$.

In this paper, we are interested in the following obstacle problem:

$$
\int_{\Omega}(A(x, d u) \cdot d(v-u)+B(x, d u) \cdot(v-u)) d x \geq 0
$$

for $v$ belonging to

$$
\begin{aligned}
\mathfrak{\Re}_{\psi, \theta}=\left\{v \in W_{d}^{p(x)}\left(\Omega, \Lambda^{l}, \mu\right): v \geq \psi,\right. \\
\text { a.e. } \left.x \in \Omega, v-\theta \in W_{0 d}^{p(x)}\left(\Omega, \Lambda^{l}, \mu\right)\right\},
\end{aligned}
$$

where $\psi(x)=\sum \psi_{I}(x) d x_{I} \in \Lambda^{l}\left(\mathbb{R}^{n}\right), v(x)=\sum v_{I}(x) d x_{I} \in$ $\Lambda^{l}\left(\mathbb{R}^{n}\right), v_{I}, \psi_{I}: \Omega \rightarrow[-\infty,+\infty] ; v \geq \psi$, a.e. $x \in \Omega$ means that, for any $I$, we have $v_{I} \geq \psi_{I}$, a.e. $x \in \Omega ; \theta \in$
$W_{d}^{p(x)}\left(\Omega, \Lambda^{l}, \mu\right), l=0,1, \ldots, n-1$, and the variable exponent $p(x) \in \mathscr{P}(\Omega)$ satisfies

$$
1<p^{-} \leq p(x) \leq p^{+}<\infty \quad \text { for a.e. } x \in \Omega \text {. }
$$

The operators $A(x, \xi): \Omega \times \Lambda^{l}\left(\mathbb{R}^{n}\right) \rightarrow \Lambda^{l}(\mathbb{R})$ and $B(x, \xi): \Omega \times \Lambda^{l}\left(\mathbb{R}^{n}\right) \rightarrow \Lambda^{l-1}(\mathbb{R})$ satisfy the following growth conditions on a bounded domain $\Omega$ :

(H1) $A(x, \xi)$ and $B(x, \xi)$ are measurable for all $\xi$ with respect to $x$ and continuous for a.e. $x \in \Omega$ with respect to $\xi$,

(H2) $|A(x, \xi)| \leq C_{1} w(x)|\xi|^{p(x)-1}$,

(H3) $A(x, \xi) \cdot \xi \geq C_{2} w(x)|\xi|^{p(x)}$,

(H4) $|B(x, \xi)| \leq C_{3} w(x)|\xi|^{p(x)-1}$,

(H5) $B(x, d \xi) \cdot \xi \geq C_{4} w(x)|\xi|^{p(x)}$

(H6) $(A(x, d \xi)-A(x, d \eta)) \cdot(d \xi-d \eta)+(B(x, d \xi)-B(x, d \eta))$. $(\xi-\eta) \geq 0$ for $\xi \neq \eta$,

where $C_{1}, C_{2}, C_{3}$, and $C_{4}$ are nonnegative constants. $w(x) \in$ $L^{1}(\Omega)$ nonnegative and $w(x)^{1 /(p(x)-1)} \in L^{1}(\Omega)$. We will discuss the existence and uniqueness of the solution $u \in \boldsymbol{\Re}_{\psi, \theta}$ for the abovementioned obstacle problem.

Now, we introduce the existing results and related definitions.

Throughout this paper, we assume that $\Omega$ is a bounded domain in $\mathbb{R}^{n}$. Let $\Lambda^{l}=\Lambda^{l}\left(\mathbb{R}^{n}\right)$ be the set of all $l$-forms in 
$\mathbb{R}^{n}$. A differential $l$-form $u(x)$ is generated by $\left\{d x_{i 1} \wedge d x_{i 2} \wedge\right.$ $\left.\cdots \wedge d x_{i l}\right\}, l=1,2, \ldots, n$; that is, $u(x)=\sum_{I} u_{I}(x) d x_{I}=$ $\sum u_{i 1, i 2, \ldots, i l}(x) d x_{i 1} \wedge d x_{i 2} \wedge \cdots \wedge d x_{i l}$, where $u_{I}(x)$ is differential function, $I=\left(i_{1}, i_{2}, \ldots, i_{l}\right)$, and $1 \leq i_{1}<i_{2}<\cdots<$ $i_{l} \leq n$. Let $D^{\prime}\left(\Omega, \Lambda^{l}\right)$ be the space of all differential $l$ forms on $\Omega$. For $\alpha(x)=\Sigma \alpha_{I}(x) d x_{I} \in \Lambda^{l}$ and $\beta(x)=$ $\Sigma \beta_{I}(x) d x_{I} \in \Lambda^{l}$, then the inner product is obtained by $\alpha \cdot \beta=\star(\alpha \wedge \star \beta)=\sum_{I} \alpha_{I}(x) \beta_{I}(x)$. We write $|u|=(u$. $u)^{1 / 2}=\left(\sum_{I}\left|u_{I}(x)\right|^{2}\right)^{1 / 2}$. We denote the exterior derivative by $d u=\sum_{i=1}^{n} \sum_{I}\left(\partial u_{I}(x) / \partial x_{i}\right) d x_{I} \wedge d x_{i}: D^{\prime}\left(\Omega, \Lambda^{l}\right) \rightarrow$ $D^{\prime}\left(\Omega, \Lambda^{l+1}\right)$ for $l=0,1, \ldots, n-1$. Its formal adjoint operator $d^{\star}: D^{\prime}\left(\Omega, \Lambda^{l+1}\right) \rightarrow D^{\prime}\left(\Omega, \Lambda^{l}\right)$ is given by $d^{\star}=(-1)^{n l+1} \star d \star$, $l=0,1,2, \ldots, n-1$; here $\star$ is the well-known Hodge star operator. Denote the class of infinitely differential $l$-forms on $\Omega$ by $C^{\infty}\left(\Omega, \Lambda^{l}\right)$. A differential $l$-form $u \in D^{\prime}\left(\Omega, \Lambda^{l}\right)$ is called a closed form if $d u=0$ in $\Omega$.

Next we will introduce some basic properties of weighted variable exponent Lebesgue spaces $L^{p(x)}(\Omega, \mu)$ and weighted variable exponent Sobolev spaces $W^{1, p(x)}(\Omega, \mu)$, and we define $\mathscr{P}(\Omega)$ to be the set of all $n$-dimensioned Lebesgue measurable functions $p: \Omega \rightarrow[1, \infty]$. Functions $p \in$ $\mathscr{P}(\Omega)$ are called variable exponents on $\Omega$. We define $p^{-}:=$ ess $\inf _{x \in \Omega} p(x), p^{+}:=$ess $\sup _{x \in \Omega} p(x)$. If $p^{+}<\infty$, then we call $p$ a bounded variable exponent. If $p \in \mathscr{P}(\Omega)$, then we define $p^{\prime} \in \mathscr{P}(\Omega)$ by $(1 / p(x))+\left(1 / p^{\prime}(x)\right)=1$, where $1 / \infty:=0$. The function $p^{\prime}$ is called the dual variable exponent of $p$. We denote $w$ as a weight if $w \in L_{\text {loc }}^{1}\left(\mathbb{R}^{n}\right)$ and $w>0$ a.e.; also in general $d \mu=w d x$. From [7, 10], we know that if $p \in \mathscr{P}(\Omega)$ satisfies (3), the weighted variable exponent Lebesgue spaces $L^{p(x)}(\Omega, \mu)=\left\{f: \int_{\Omega}|\lambda f(x)|^{p(x)} d \mu<\right.$ $\infty, \lambda>0\}$ with the norm $\|f\|_{L^{p(x)}(\Omega, \mu)}=\inf \{\lambda>0$ : $\left.\int_{\Omega}|f(x) / \lambda|^{p(x)} d \mu \leq 1\right\}$ and the weighted variable exponent Sobolev spaces $W^{1, p(x)}(\Omega, \mu)=\left\{f \in L^{p(x)}(\Omega, \mu): \nabla f \in\right.$ $\left.L^{p(x)}(\Omega, \mu)\right\}$ with the norm $\|f\|_{W^{1, p(x)}(\Omega, \mu)}=\|f\|_{L^{p(x)}(\Omega, \mu)}+$ $\|\nabla f\|_{L^{p(x)}(\Omega, \mu)}$ are Banach space and reflexive and uniformly convex. On the set of all differential forms on $\Omega$, we define the weighted variable exponent Lebesgue spaces of differential $l$-forms $L^{p(x)}\left(\Omega, \Lambda^{l}, \mu\right)$ and the weighted variable exponent Sobolev spaces of differential forms $W_{d}^{p(x)}\left(\Omega, \Lambda^{l}, \mu\right)$.

Definition 1. We denote the weighted variable exponent Lebesgue spaces of differential $l$-forms by $L^{p(x)}\left(\Omega, \Lambda^{l}, \mu\right)=$ $\left\{u=\sum_{I} u_{I}(x) d x_{I} \in \Lambda^{l}: u_{I}(x) \in L^{p(x)}(\Omega, \mu)\right\} l=0,1,2, \ldots, n$ and we endow $L^{p(x)}\left(\Omega, \Lambda^{l}, \mu\right)$ with the following norm:

$$
\|u\|_{L^{p(x)}\left(\Omega, \Lambda^{l}, \mu\right)}=\inf \left\{\lambda>0: \int_{\Omega}\left|\frac{u}{\lambda}\right|^{p(x)} d \mu \leq 1\right\} .
$$

And the spaces $W_{d}^{p(x)}\left(\Omega, \Lambda^{l}, \mu\right)=\left\{u \in L^{p(x)}\left(\Omega, \Lambda^{l}, \mu\right): d u \in\right.$ $\left.L^{p(x)}\left(\Omega, \Lambda^{l+1}, \mu\right)\right\}$ with the norm

$$
\|u\|_{W_{d}^{p(x)}\left(\Omega, \Lambda^{1}, \mu\right)}=\|u\|_{L^{p(x)}\left(\Omega, \Lambda^{l}, \mu\right)}+\|d u\|_{L^{p(x)}\left(\Omega, \Lambda^{l+1}, \mu\right)}
$$

are the weighted variable exponent Sobolev spaces of differential $l$-forms; $l=0,1,2, \ldots, n-1 . W_{0 d}^{p(x)}\left(\Omega, \Lambda^{l}, \mu\right)$ is the completion of $C_{0}^{\infty}\left(\Omega, \Lambda^{l}, \mu\right)$ in $W_{d}^{p(x)}\left(\Omega, \Lambda^{l}, \mu\right)$. We need the following Hölder inequalities; see $[7,10]$.

Proposition 2. Let $p, q \in \mathscr{P}(\Omega)$ be such that $1=(1 / p(x))+$ $(1 / q(x))$ for $\mu$-almost every $x \in \Omega$. Then

$$
\begin{aligned}
& \int_{\Omega}|f g| d \mu \leq \int_{\Omega} \frac{1}{p(x)}|f|^{p(x)} d \mu+\int_{\Omega} \frac{1}{q(x)}|g|^{q(x)} d \mu, \\
& \int_{\Omega}|f g| d \mu \leq\left(\left(\frac{1}{p}\right)^{+}+\left(\frac{1}{q}\right)^{+}\right)\|f\|_{L^{p(x)}(\Omega, \mu)}\|g\|_{L^{q(x)}(\Omega, \mu)^{\prime}}
\end{aligned}
$$

for all $f \in L^{p(x)}(\Omega, \mu)$ and $g \in L^{q(x)}(\Omega, \mu)$.

Lemma 3 (see [7]). Let $\left(D, \sum, \mu\right)$ be a $\sigma$-finite, complete measure space; if $f \in L^{p(\cdot)}(D, \mu), g \in L^{0}(D, \mu)$, and $0 \leq$ $|g| \leq|f| \mu$-almost everywhere, then $g \in L^{p(\cdot)}(D, \mu)$ and $\|g\|_{L^{p(x)}(D, \mu)} \leq\|f\|_{L^{p(x)}(D, \mu)}$.

By the inequality $\left(\sum_{i=1}^{n}\left(a_{i}\right)^{2}\right)^{1 / 2} \leq \sum_{i=1}^{n} a_{i} \leq$ $n^{1 / 2}\left(\sum_{i=1}^{n}\left(a_{i}\right)^{2}\right)^{1 / 2}$ for any $a_{i} \geq 0$, using Lemma 3 , we can easily have the following lemma.

Lemma 4. If $u=\Sigma_{I} u_{I}(x) d x_{I} \in D^{\prime}\left(\Omega, \Lambda^{l}\right)$ and $|u|=$ $\left(\sum_{I}\left|u_{I}\right|^{2}\right)^{1 / 2}$, then $u \in L^{p(x)}\left(\Omega, \Lambda^{l}, \mu\right)$ and $|u| \in L^{p(x)}(\Omega, \mu)$ are equivalent, and $\|u\|_{L^{p(x)}\left(\Omega, \Lambda^{l}, \mu\right)}=\||u|\|_{L^{p(x)}(\Omega, \mu)}$.

\section{Main Results}

In this section, we will obtain the existence and uniqueness of weak solution for obstacle problem of the nonhomogeneous $A$-harmonic equation in space $W_{d}^{p(x)}\left(\Omega, \Lambda^{l}, \mu\right)$.

Theorem 5. Suppose $\mathfrak{\Re}_{\psi, \theta}$ is not empty, under conditions (H1)-(H6), and there exists a unique solution $u$ to the obstacle problem (1)-(2). That is, there is a differential form $u$ in $\boldsymbol{\Omega}_{\psi, \theta}$ such that

$$
\int_{\Omega}(A(x, d u) \cdot d(v-u)+B(x, d u) \cdot(v-u)) d x \geq 0,
$$

whenever $v \in \boldsymbol{\Omega}_{\psi, \theta}$.

We deduce Theorem 5 from a proposition of Kinderlehrer and Stampacchia.

Proposition 6 (see [12]). Let $K$ be a nonempty closed convex subset of $X$ and let $\mathfrak{A}: K \rightarrow X^{\prime}$ be monotone, coercive, and weakly continuous on $K$. Then there exists an element $u$ in $K$ such that $\langle\mathfrak{\mathcal { A }} u, v-u\rangle \geq 0$ whenever $v \in K$.

Now let $X=W_{d}^{p(x)}\left(\Omega, \Lambda^{l}, \mu\right)$ and $\langle\cdot, \cdot\rangle$ be the usual pairing between $X$ and $X^{\prime},\langle f, g\rangle=\int_{\Omega} f \cdot g d \mu$, where $g$ is in $X$ and $f$ in $X^{\prime}=W_{d}^{p^{\prime}(x)}\left(\Omega, \Lambda^{l}, \mu\right)$. We will take $\Omega_{\psi, \theta}$ as $K$. We define a mapping $\mathfrak{A}: \mathfrak{\Omega}_{\psi, \theta} \rightarrow X^{\prime}$ by

$$
\langle\mathfrak{U} v, u\rangle=\int_{\Omega}(A(x, d v) \cdot d u+B(x, d v) \cdot u) d x
$$

for $u \in W_{d}^{p^{(x)}}\left(\Omega, \Lambda^{l}, \mu\right)$. 
Lemma 7. If $p(x)$ satisfies (3), then spaces $L^{p(x)}\left(\Omega, \Lambda^{l}, \mu\right)$ and $W_{d}^{p(x)}\left(\Omega, \Lambda^{l}, \mu\right)$ are complete and convex.

Proof. From [7], we know that if $p$ satisfies (3) and $w(x)^{1 /(p(x)-1)} \in L^{1}(\Omega)$, then let $L^{p(x)}(\Omega, \mu)$ be Banach space and uniformly convex. If $\omega_{1}$ and $\omega_{2}$ are two $l$-forms: $\omega_{1}=$ $\sum_{I} a_{I} d x_{I}$ and $\omega_{2}=\sum_{I} b_{I} d x_{I}$, we can easily have $\omega_{1}+\omega_{2}=$ $\sum_{I}\left(a_{I}+b_{I}\right) d x_{I}$ and $d\left(\omega_{1}+\omega_{2}\right)=d \omega_{1}+d \omega_{2}$, so we can immediately obtain the convexity of spaces $L^{p(x)}\left(\Omega, \Lambda^{l}, \mu\right)$ and $W_{d}^{p(x)}\left(\Omega, \Lambda^{l}, \mu\right)$.

Let $u_{j}=\sum_{I} u_{j I} d x_{I} \in L^{p(x)}\left(\Omega, \Lambda^{l}, \mu\right)$ be a Cauchy sequence in $W_{d}^{p(x)}\left(\Omega, \Lambda^{l}, \mu\right)$. Then for any $I, u_{j I}(x)$ converges in $L^{p(x)}(\Omega, \mu)$. Suppose that $u_{j I}(x) \rightarrow u_{I}(x)$ in $L^{p(x)}(\Omega, \mu)$. Now let $u=\sum_{I} u_{I} d x_{I} \in L^{p(x)}\left(\Omega, \Lambda^{l}, \mu\right)$, we have

$$
\left|u_{j}-u\right|=\left(\sum_{I}\left|u_{j I}-u_{I}\right|^{2}\right)^{1 / 2} \leq \sum_{I}\left|u_{j I}-u_{I}\right|
$$

using Lemmas 3 and 4 , and we know the sequence $u_{j}$ converges to $u$ in $L^{p(x)}\left(\Omega, \Lambda^{l}, \mu\right)$.

For the sequence $\left\{d u_{j}\right\}$, we suppose $d u_{j} \rightarrow \quad v$ in $L^{p(x)}\left(\Omega, \Lambda^{l+1}, \mu\right)$, and then $v \in L^{p(x)}\left(\Omega, \Lambda^{l+1}, \mu\right)$. So $\left(u_{j}, d u_{j}\right)$ converges to $(u, v)$ in the normed space $L^{p(x)}\left(\Omega, \Lambda^{l}, \mu\right) \times$ $L^{p(x)}\left(\Omega, \Lambda^{l+1}, \mu\right)$. From $d u_{j}-d u=\sum_{I}\left(d u_{j I}-d u_{I}\right) \wedge d x_{I}$, we have

$$
\begin{aligned}
\left|d u_{j}-d u\right| & =\left|\sum_{I} \sum_{i=1}^{n} \frac{\partial u_{j I}-\partial u_{I}}{\partial x_{i}} d x_{i} \wedge d x_{I}\right| \\
& \leq\left(\sum_{I} \sum_{i=1}^{n}\left|\frac{\partial u_{j I}-\partial u_{I}}{\partial x_{i}}\right|^{2}\right)^{1 / 2} \\
& \leq \sum_{I} \sum_{i=1}^{n}\left|\frac{\partial u_{j I}-\partial u_{I}}{\partial x_{i}}\right| \\
& \leq \sum_{I} n^{1 / 2}\left(\sum_{i=1}^{n}\left|\frac{\partial u_{j I}-\partial u_{I}}{\partial x_{i}}\right|^{2}\right)^{1 / 2} \\
& =n^{1 / 2} \sum_{I}\left|\nabla u_{j I}-\nabla u_{I}\right| .
\end{aligned}
$$

In view of [10], for any $I$, we know that $u_{j I} \rightarrow u_{I}$ in $L^{p(x)}(\Omega, \mu)$ and $\nabla u_{j I} \rightarrow v_{I}$ in $L^{p(x)}(\Omega, \mu)$, and then $\nabla u_{I}=v_{I} \in L^{p(x)}(\Omega, \mu)$. Using Lemmas 3 and 4 , we get the sequence $d u_{j}$ that converges to $d u$ in $L^{p(x)}\left(\Omega, \Lambda^{l}, \mu\right)$; it follows that $v=d u \in L^{p(x)}\left(\Omega, \Lambda^{l+1}, \mu\right)$. So, we prove $W_{d}^{p(x)}\left(\Omega, \Lambda^{l}, \mu\right)$ is a closed subspace of $L^{p(x)}\left(\Omega, \Lambda^{l}, \mu\right) \times$ $L^{p(x)}\left(\Omega, \Lambda^{l+1}, \mu\right)$. This ends the proof of Lemma 7 . lemma.

Using Lemma 7, we can immediately obtain the following

Lemma 8. $\mathfrak{\Re}_{\psi, \theta}$ is a closed convex set.
Lemma 9. For each $v \in \mathfrak{\Re}_{\psi, \theta}, \mathfrak{A} v \in\left[W_{d}^{p(x)}\left(\Omega, \Lambda^{l}, \mu\right)\right]^{\prime}$.

Proof. Using Hölder inequality (7) with $1=(1 / p(x))+$ $\left(1 / p^{\prime}(x)\right)$ and $(\mathrm{H} 2)$, we get

$$
\begin{aligned}
& \left|\int_{\Omega} A(x, v(x)) \cdot u(x) d x\right| \\
& \quad \leq C_{1} \int_{\Omega}|v(x)|^{p(x)-1}|u(x)| w(x) d x \\
& \quad \leq C_{1}\left(\frac{1}{p^{-}}+\frac{1}{p^{\prime-}}\right)\left\||v(x)|^{p(x)-1}\right\|_{L^{p^{\prime}(x)}\left(\Omega, \Lambda^{l}, \mu\right)}\|u\|_{L^{p(x)}\left(\Omega, \Lambda^{l}, \mu\right)} \\
& \quad \leq 2 C_{1}\|v(x)\|_{L^{p(x)}\left(\Omega, \Lambda^{l}, \mu\right)}^{p(x)}\|u\|_{L^{p(x)}\left(\Omega, \Lambda^{l}, \mu\right)} .
\end{aligned}
$$

Similarly, for the operator $B(x, \xi)$, we have

$$
\begin{aligned}
& \left|\int_{\Omega} B(x, v(x)) \cdot u(x) d x\right| \\
& \quad \leq 2 C_{3}\|v(x)\|_{L^{p(x)}\left(\Omega, \Lambda^{l}, \mu\right)}^{p(x)-1}\|u\|_{L^{p(x)}\left(\Omega, \Lambda^{l}, \mu\right)} .
\end{aligned}
$$

Using (12) and (13) we can easily prove

$$
\begin{aligned}
& |\langle\hat{\mathfrak{A}} v, u\rangle| \\
& =\left|\int_{\Omega}(A(x, d v) \cdot d u+B(x, d v) \cdot u) d x\right| \\
& \leq 2 C_{1}\|d v\|_{L^{p(x)}\left(\Omega, \Lambda^{l+1}, \mu\right)}^{p(x)-1}\|d u\|_{L^{p(x)}\left(\Omega, \Lambda^{l+1}, \mu\right)} \\
& \quad+2 C_{3}\|d v\|_{L^{p(x)}\left(\Omega, \Lambda^{l+1}, \mu\right)}^{p(x)-1}\|u\|_{L^{p(x)}\left(\Omega, \Lambda^{l}, \mu\right)} \\
& \leq C_{5}\|d v\|_{L^{p(x)}\left(\Omega, \Lambda^{l+1}, \mu\right)}^{p(x)-1}\left(\|d u\|_{L^{p(x)}\left(\Omega, \Lambda^{l+1}, \mu\right)}+\|u\|_{L^{p(x)}\left(\Omega, \Lambda^{l}, \mu\right)}\right) \\
& \leq C_{5}\|v(x)\|_{W_{d}^{p(x)}\left(\Omega, \Lambda^{l}, \mu\right)}^{p(x)-1}\|u\|_{W_{d}^{p(x)}\left(\Omega, \Lambda^{l}, \mu\right)} .
\end{aligned}
$$

So we get $\mathfrak{A} v \in\left[W_{d}^{p(x)}\left(\Omega, \Lambda^{l}, \mu\right)\right]^{\prime}$, whenever $v \in \mathfrak{\Re}_{\psi, \theta}, u \in$ $W_{d}^{p(x)}\left(\Omega, \Lambda^{l}, \mu\right)$, and $C_{1}$ and $C_{3}$ are constants fixed to (H2) and (H4).

Lemma 10. $\mathfrak{A}$ is monotone and coercive on $\mathfrak{\Re}_{\psi, \theta}$. 
Proof. It follows from (H6) that $\mathfrak{A}$ is monotone. To show that $\mathfrak{A}$ is coercive on $\mathfrak{R}_{\psi, \theta}$, fix $\varphi \in \mathfrak{\Re}_{\psi, \theta}$ and using the conditions (H2)-(H5), (12), (13), and (6), then

$$
\begin{aligned}
& \langle\mathfrak{\mathcal { Y }} u-\mathfrak{A} \varphi, u-\varphi\rangle \\
& =\int_{\Omega}((A(x, d u)-A(x, d \varphi)) \cdot(d u-d \varphi) \\
& +(B(x, d u)-B(x, d \varphi)) \cdot(u-\varphi)) d x \\
& =\int_{\Omega}(A(x, d u) \cdot d u) d x+\int_{\Omega}(A(x, d \varphi) \cdot d \varphi) d x \\
& -\int_{\Omega}(A(x, d u) \cdot d \varphi) d x-\int_{\Omega}(A(x, d \varphi) \cdot d u) d x \\
& +\int_{\Omega}(B(x, d u) \cdot u) d x+\int_{\Omega}(B(x, d \varphi) \cdot \varphi) d x \\
& -\int_{\Omega}(B(x, d u) \cdot \varphi) d x-\int_{\Omega}(B(x, d \varphi) \cdot u) d x \\
& \geq C_{2}\left(\int_{\Omega}|d u|^{p(x)} w(x) d x+\int_{\Omega}|d \varphi|^{p(x)} w(x) d x\right) \\
& -C_{1} \int_{\Omega}|d u|^{p(x)-1}|d \varphi| w(x) d x-2 C_{1}\|d \varphi\|^{p(x)-1}\|d u\| \\
& +C_{4}\left(\int_{\Omega}|u|^{p(x)} w(x) d x+\int_{\Omega}|\varphi|^{p(x)} w(x) d x\right) \\
& -C_{3} \int_{\Omega}|d u|^{p(x)-1}|\varphi| w(x) d x-2 C_{3}\|d \varphi\|^{p(x)-1}\|u\| \\
& \geq C_{2} \int_{\Omega}|d u|^{p(x)} d \mu-C_{1} \int_{\Omega} \frac{1}{p^{\prime}(x)} \varepsilon|d u|^{p(x)} d \mu \\
& -C_{1} \int_{\Omega} \frac{1}{p(x)} \varepsilon^{1-p(x)}|d \varphi|^{p(x)} d \mu \\
& -C_{3} \int_{\Omega} \frac{1}{p^{\prime}(x)} \varepsilon|d u|^{p(x)} d \mu \\
& -C_{3} \int_{\Omega} \frac{1}{p(x)} \varepsilon^{1-p(x)}|\varphi|^{p(x)} d \mu+C_{4} \int_{\Omega}|u|^{p(x)} d \mu \\
& -2 \max \left(C_{1}, C_{3}\right)\|d \varphi\|^{p(x)-1}(\|d u\|+\|u\|) \\
& +C\left(\varphi, p(x), C_{2}, C_{4}\right) \text {, }
\end{aligned}
$$

where $\|\cdot\|$ is the $L^{p(x)}\left(\Omega, \Lambda^{l}, \mu\right)$ norm; taking $\varepsilon=$ $C_{2}\left(p^{\prime}\right)^{-} / 2\left(C_{1}+C_{3}\right)$, we have

$$
\begin{aligned}
\langle\mathfrak{A} u & -\mathfrak{A} \varphi, u-\varphi\rangle \\
\geq & \frac{C_{2}}{2} \int_{\Omega}|d u|^{p(x)} d \mu+C_{4} \int_{\Omega}|u|^{p(x)} d \mu \\
& \quad-C_{5}\|d \varphi\|^{p(x)-1}(\|d u\|+\|u\|)+C_{6}
\end{aligned}
$$

$$
\begin{aligned}
& \geq \frac{C_{2}}{2} \int_{\Omega}\left(|d u-d \varphi|^{p(x)}+|d \varphi|^{p(x)}\right) d \mu \\
& \quad+C_{4} \int_{\Omega}\left(|u-\varphi|^{p(x)}+|\varphi|^{p(x)}\right) d \mu \\
& \quad-C_{5}\|d \varphi\|^{p(x)-1}(\|d u-d \varphi\|+\|d \varphi\| \\
& \quad+\|u-\varphi\|+\|\varphi\|)+C_{6} \\
& \geq C_{7}\left(\int_{\Omega}|d u-d \varphi|^{p(x)} d \mu+\int_{\Omega}|u-\varphi|^{p(x)} d \mu\right) \\
& \quad-C_{5}\|d \varphi\|^{p(x)-1}(\|d u-d \varphi\|+\|u-\varphi\|)+C_{8} .
\end{aligned}
$$

Let $\rho_{p(\cdot)}(t)=\int_{\Omega}|t|^{p(x)} d \mu$; from [7, pages 24, 73], we know that if the variable exponent $p \in \mathscr{P}(\Omega)$ satisfied $p^{+}<\infty$, then

$$
\begin{aligned}
& \min \left\{\left(\varrho_{p(\cdot)}(f)\right)^{1 / p^{-}},\left(\varrho_{p(\cdot)}(f)\right)^{1 / p^{+}}\right\} \\
& \leq\|f\|_{L^{p(x)}(\omega, \mu)} \leq \max \left\{\left(\varrho_{p(\cdot)}(f)\right)^{1 / p^{-}},\left(\varrho_{p(\cdot)}(f)\right)^{1 / p^{+}}\right\}
\end{aligned}
$$

holds. Whenever $\|u\|_{W_{d}^{p(x)}\left(\Omega, \Lambda^{l}, \mu\right)} \rightarrow \infty$, we have $\|d u-d \varphi\|_{L^{p(x)}\left(\Omega, \Lambda^{l+1}, \mu\right)}>1$ or $\|u-\varphi\|_{L^{p(x)}\left(\Omega, \Lambda^{l}, \mu\right)}>1$, and using (17) we have

$$
\begin{gathered}
\int_{\Omega}|d u-d \varphi|^{p(x)} d \mu+\int_{\Omega}|u-\varphi|^{p(x)} d \mu \\
\geq \max \left\{\|d u-d \varphi\|^{p^{-}},\|d u-d \varphi\|^{p^{+}}\right\} \\
+\max \left\{\|u-\varphi\|^{p^{-}},\|u-\varphi\|^{p^{+}}\right\} .
\end{gathered}
$$

Substituting (18) in (16) we obtain

$$
\begin{aligned}
&\langle\mathfrak{P} u-\mathfrak{A} \varphi, u-\varphi\rangle \\
& \geq C_{7}\left(\int_{\Omega}|d u-d \varphi|^{p(x)} d \mu+\int_{\Omega}|u-\varphi|^{p(x)} d \mu\right) \\
&-C_{5}\|d \varphi\|^{p(x)-1}(\|d u-d \varphi\|+\|u-\varphi\|)+C_{8} \\
& \geq C_{9}\left(\max \left\{\|d u-d \varphi\|^{p^{-}},\|d u-d \varphi\|^{p^{+}}\right\}\right. \\
&\left.\quad+\max \left\{\|u-\varphi\|^{p^{-}},\|u-\varphi\|^{p^{+}}\right\}\right) \\
&-C_{5}\|d \varphi\|^{p(x)-1}(\|d u-d \varphi\|+\|u-\varphi\|)+C_{8} .
\end{aligned}
$$

Then it is easy to obtain

$$
\frac{\langle\mathfrak{A} u-\mathfrak{A} \varphi, u-\varphi\rangle}{\|u-\varphi\|_{W_{d}^{p(x)}\left(\Omega, \Lambda^{l}, \mu\right)}} \longrightarrow \infty
$$

as $\|u-\varphi\|_{W_{d}^{p(x)}\left(\Omega, \Lambda^{l}, \mu\right)} \rightarrow \infty$. It follows that $\mathfrak{\mathcal { I }}$ is coercive on $\boldsymbol{\Re}_{\psi, \theta}$. This completes the proof of Lemma 10. 
Lemma 11. $\mathfrak{A}$ is weakly continuous on $\mathfrak{R}_{\psi, \theta}$.

Proof. Let $u_{i} \in \boldsymbol{\Omega}_{\psi, \theta}$ be a sequence that converges to an element $u \in \mathfrak{\Re}_{\psi, \theta}$ in $W_{d}^{p(x)}\left(\Omega, \Lambda^{l}, \mu\right)$. Pick a subsequence $u_{i j}$ such that $u_{i j} \rightarrow u$ a.e. in $\Omega$. Since the mapping $\xi \rightarrow A(x, \xi)$ and $\xi \rightarrow B(x, \xi)$ are continuous for a.e. $x$ in $\Omega$, we have $A\left(x, u_{i j}(x)\right) w^{-1 / p(x)} \rightarrow A(x, u(x)) w^{-1 / p(x)}$ a.e. in $\Omega$. Under the conditions (H2) and (H4), we know that $A\left(x, d u_{i j}\right) w^{-1 / p(x)}$ and $B\left(x, d u_{i j}\right) w^{-1 / p(x)}$ are uniformly bounded in $L^{p(x)}\left(\Omega, \Lambda^{l+1}\right)$, and we have $A\left(x, d u_{i j}\right) w^{-1 / p(x)} \quad \rightarrow \quad A(x, d u) w^{-1 / p(x)}$ weakly in $L^{p(x)}\left(\Omega, \Lambda^{l+1}, \mu\right)$ and $B\left(x, d u_{i j}\right) w^{-1 / p(x)} \rightarrow B\left(x, d u_{i j}\right) w^{-1 / p(x)}$ weakly in $L^{p(x)}\left(\Omega, \Lambda^{l}, \mu\right)$.

Since the weak limit is independent of the choice of the subsequence, it follows that

$$
\begin{aligned}
& A\left(x, d u_{i}\right) w^{-1 / p(x)} \longrightarrow A(x, d u) w^{-1 / p(x)} \\
& B\left(x, d u_{i}\right) w^{-1 / p(x)} \longrightarrow B(x, d u) w^{-1 / p(x)}
\end{aligned}
$$

for all $u \in L^{p(x)}\left(\Omega, \Lambda^{l}, \mu\right), d u w^{1 / p(x)} \in L^{p(x)}\left(\Omega, \Lambda^{l+1}\right)$.

Then we have

$$
\begin{aligned}
& \left\langle\mathfrak{\mathcal { A }} u_{i}, v\right\rangle \\
& =\int_{\Omega}\left(A\left(x, d u_{i}\right) w^{-1 / p(x)} \cdot d v w^{1 / p(x)}\right) d x \\
& \quad+\int_{\Omega}\left(B\left(x, d u_{i}\right) w^{-1 / p(x)} \cdot v w^{1 / p(x)}\right) d x \\
& \longrightarrow \int_{\Omega}\left(A(x, d u) w^{-1 / p(x)} \cdot d v w^{1 / p(x)}\right) d x \\
& \quad+\int_{\Omega}\left(B(x, d u) w^{-1 / p(x)} \cdot v w^{1 / p(x)}\right) d x=\langle\mathfrak{Y} u, v\rangle .
\end{aligned}
$$

Hence $\mathfrak{A}$ is weakly continuous on $\boldsymbol{\Re}_{\psi, \theta}$. This ends the proof of Lemma 11.

Proof of Theorem 5. We can apply Proposition 6 and the above lemmas to obtain the existence. If there are two weak solutions $u_{1}, u_{2} \in \mathfrak{R}_{\psi, \theta}$ to obstacle problem (1)-(2), then we have

$$
\begin{aligned}
& \int_{\Omega}\left(A\left(x, d u_{1}\right) \cdot d\left(u_{2}-u_{1}\right)+B\left(x, d u_{1}\right) \cdot\left(u_{2}-u_{1}\right)\right) d x \geq 0 \\
& \int_{\Omega}\left(A\left(x, d u_{2}\right) \cdot d\left(u_{1}-u_{2}\right)+B\left(x, d u_{2}\right) \cdot\left(u_{1}-u_{2}\right)\right) d x \geq 0
\end{aligned}
$$

so

$$
\begin{aligned}
& \int_{\Omega}\left(\left(A\left(x, d u_{2}\right)-A\left(x, d u_{1}\right)\right) \cdot d\left(u_{2}-u_{1}\right)\right. \\
& \left.\quad+\left(B\left(x, d u_{2}\right)-B\left(x, d u_{1}\right)\right) \cdot\left(u_{2}-u_{1}\right)\right) d x \leq 0 .
\end{aligned}
$$

In view of (H6), we can further infer that

$$
\begin{gathered}
\int_{\Omega}\left(\left(A\left(x, d u_{2}\right)-A\left(x, d u_{1}\right)\right) \cdot d\left(u_{2}-u_{1}\right)\right. \\
+\left(B\left(x, d u_{2}\right)-B\left(x, d u_{1}\right)\right) \\
\left.\cdot\left(u_{2}-u_{1}\right)\right) d x=0 \quad \text { a.e. on } \Omega,
\end{gathered}
$$

which means that $u_{1}=u_{2}$ a.e. on $\Omega$, and now we complete the proof.

Corollary 12. Let $\Omega$ be a bounded domain and $\theta \in$ $W_{d}^{p(x)}\left(\Omega, \Lambda^{l-1}, \mu\right)$. Under the conditions (H1)-(H6), there is a differential form $u \in W_{d}^{p(x)}\left(\Omega, \Lambda^{l-1}, \mu\right)$ with $u-\theta \in$ $W_{d}^{p(x)}\left(\Omega, \Lambda^{l-1}, \mu\right)$ such that

$$
d^{\star} A(x, d u)=B(x, d u), \quad \text { weakly in } \Omega ;
$$

that is, $\int_{\Omega}(A(x, d u) \cdot d \varphi+B(x, d u) \cdot \varphi) d x=0$, whenever $\varphi \epsilon$ $W_{0 d}^{p(x)}\left(\Omega, \Lambda^{l-1}, \mu\right), l=1,2, \ldots, n$.

Proof. Choose $\psi_{I} \equiv-\infty$ and let $u$ be the solution to the obstacle problem (1)-(2) in $\Re_{\psi, \theta}$. For any $\varphi \in W_{0 d}^{p^{(x)}}\left(\Omega, \Lambda^{l-1}, \mu\right)$, since $u+\varphi$ and $u-\varphi$ both belong to $\mathfrak{R}_{\psi, \theta}$, we have

$$
\begin{gathered}
-\left(\int_{\Omega}(A(x, d u) \cdot d \varphi+B(x, d u) \cdot \varphi) d x\right) \geq 0, \\
\int_{\Omega}(A(x, d u) \cdot d \varphi+B(x, d u) \cdot \varphi) d x \geq 0 .
\end{gathered}
$$

Thus

$$
\int_{\Omega}(A(x, d u) \cdot d \varphi+B(x, d u) \cdot \varphi) d x=0
$$

as desired.

Remark 13. If $p(x)=p$, then $\|u\|_{L^{p(x)}\left(\Omega, \Lambda^{l}, \mu\right)}=\|u\|_{L^{p}\left(\Omega, \Lambda^{l}, \mu\right)}=$ $\|u\|_{p, \Omega, \mu}=\left(\int_{\Omega}|u|^{p} d \mu\right)^{1 / p}$ and the Luxemburg norm reduces to the $L^{p}$ norm. So (26) is the extension of the equation in [2-5].

\section{Conflict of Interests}

The author declares that there is no conflict of interests regarding the publication of this paper.

\section{References}

[1] R. P. Agarwal and S. Ding, "Advances in differential forms and the A-harmonic equation," Mathematical and Computer Modelling, vol. 37, no. 12-13, pp. 1393-1426, 2003.

[2] S. Ding, "Two-weight Caccioppoli inequalities for solutions of nonhomogeneous $A$-harmonic equations on Riemannian manifolds," Proceedings of the American Mathematical Society, vol. 132, no. 8, pp. 2367-2375, 2004.

[3] S. Ding and C. A. Nolder, "Weighted Poincaré inequalities for solutions to A-harmonic equations," Illinois Journal of Mathematics, vol. 46, no. 1, pp. 199-205, 2002. 
[4] H. Wen, "Weighted norm inequalities for solutions to the nonhomogeneous A-harmonic equation," Journal of Inequalities and Applications, vol. 2009, Article ID 851236, 2009.

[5] H. Wen, "The integral estimate with Orlicz norm in $L^{\varphi(x)}$ averaging domain," Journal of Inequalities and Applications, vol. 2011, article 90, 2011.

[6] Z. H. Cao, G. J. Bao, and H. J. Zhu, "The obstacle problem for the A-harmonic equation," Journal of Inequalities and Applications, vol. 2010, Article ID 767150, 2010.

[7] L. Diening, P. Harjulehto, P. Hästö, and M. Růžička, Lebesgue and Sobolev Spaces with Variable Exponents, vol. 2017 of Lecture Notes in Mathematics, Springer, Heidelberg, Germany, 2011.

[8] S. Samko and B. Vakulov, "Weighted Sobolev theorem with variable exponent for spatial and spherical potential operators," Journal of Mathematical Analysis and Applications, vol. 310, no. 1, pp. 229-246, 2005.

[9] D. Cruz-Uribe, A. Fiorenza, and C. J. Neugebauer, "Weighted norm inequalities for the maximal operator on variable Lebesgue spaces," Journal of Mathematical Analysis and Applications, vol. 394, no. 2, pp. 744-760, 2012.

[10] F. Yongqiang, "Weak solution for obstacle problem with variable growth," Nonlinear Analysis: Theory, Methods \& Applications, vol. 59, no. 3, pp. 371-383, 2004.

[11] J. F. Rodrigues, M. Sanchón, and J. M. Urbano, “The obstacle problem for nonlinear elliptic equations with variable growth and $L^{1}$-data," Monatshefte für Mathematik, vol. 154, no. 4, pp. 303-322, 2008.

[12] D. Kinderlehrer and G. Stampacchia, An Introduction to Variational Inequalities and Their Applications, vol. 88 of Pure and Applied Mathematics, Academic Press, New York, NY, USA, 1980. 


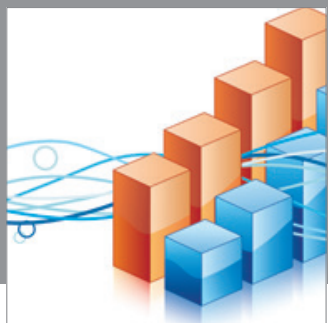

Advances in

Operations Research

mansans

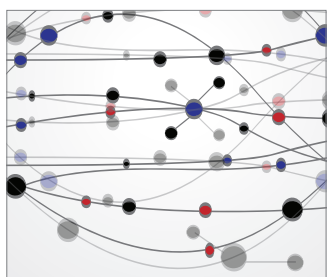

The Scientific World Journal
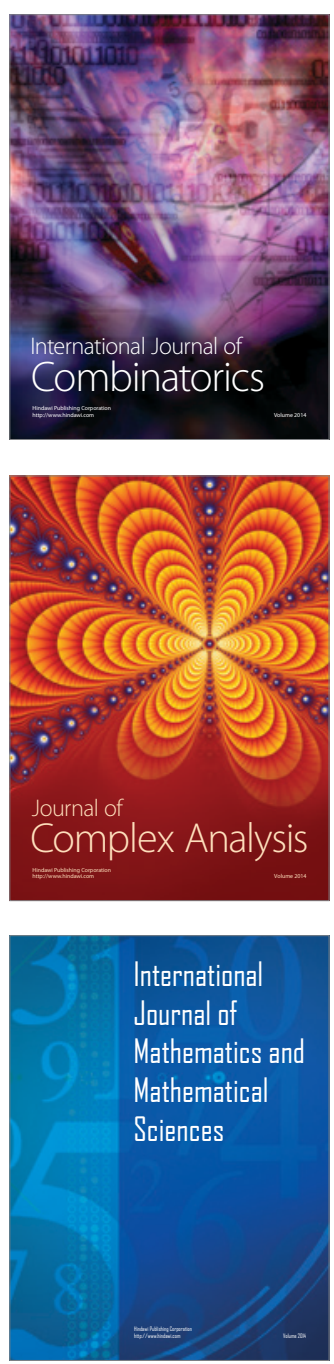
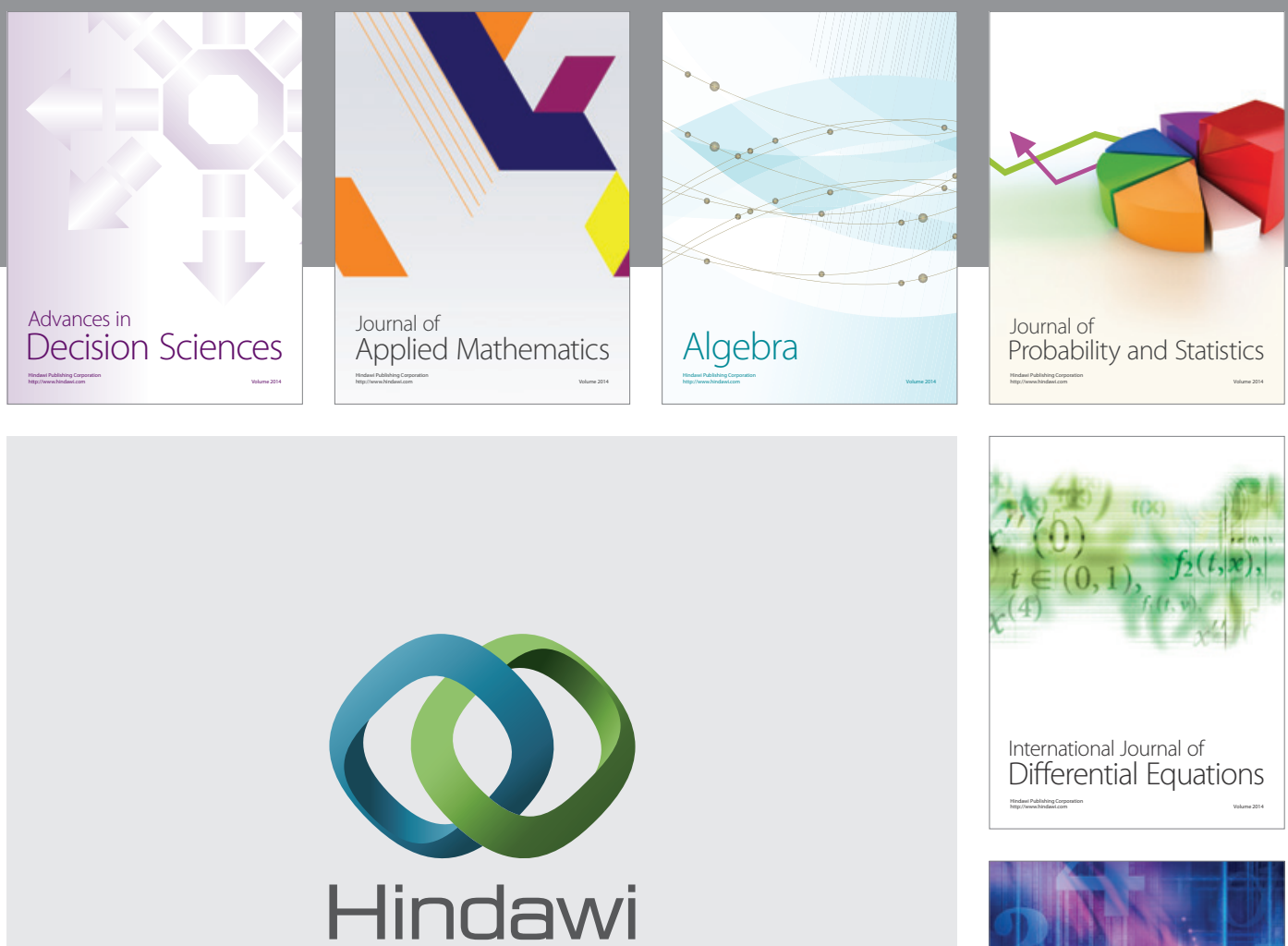

Submit your manuscripts at http://www.hindawi.com
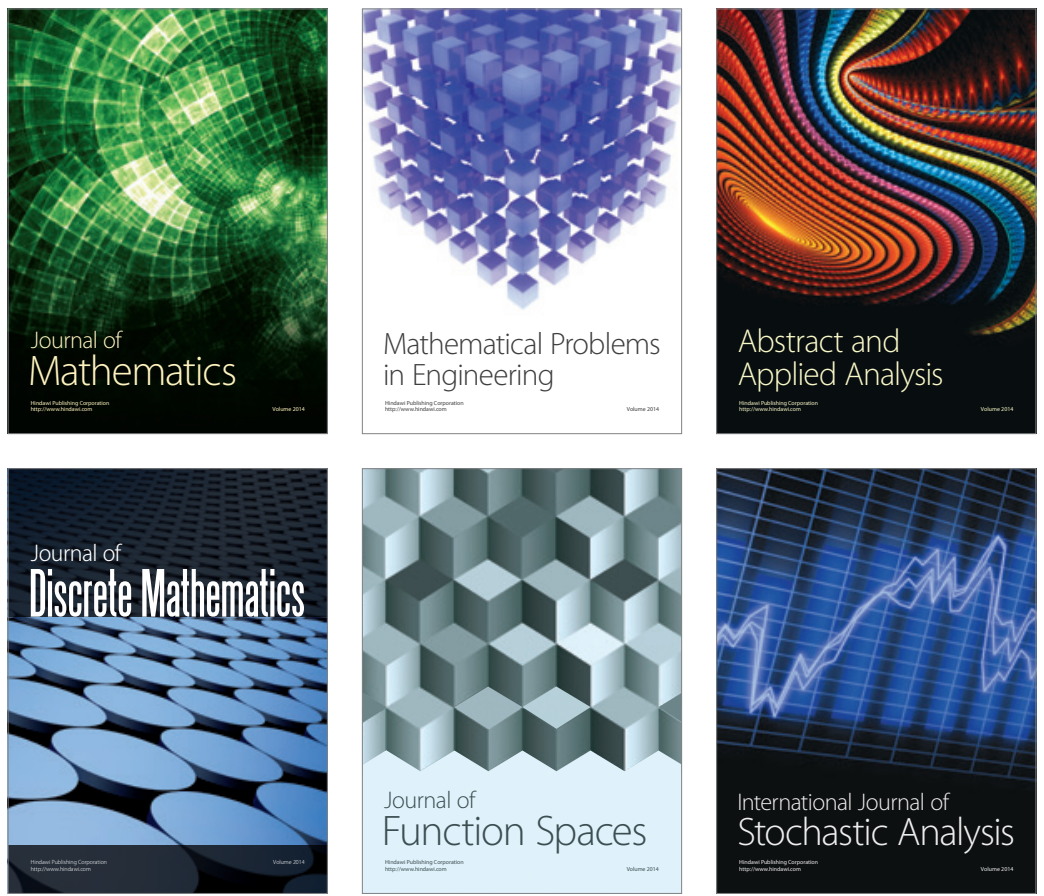

Journal of

Function Spaces

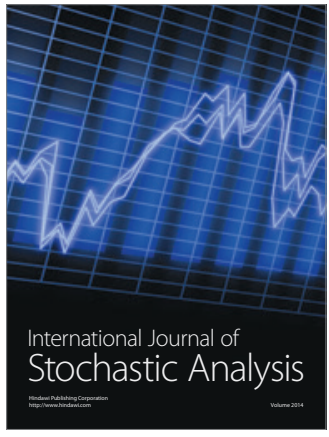

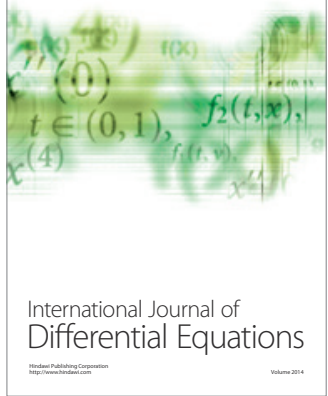
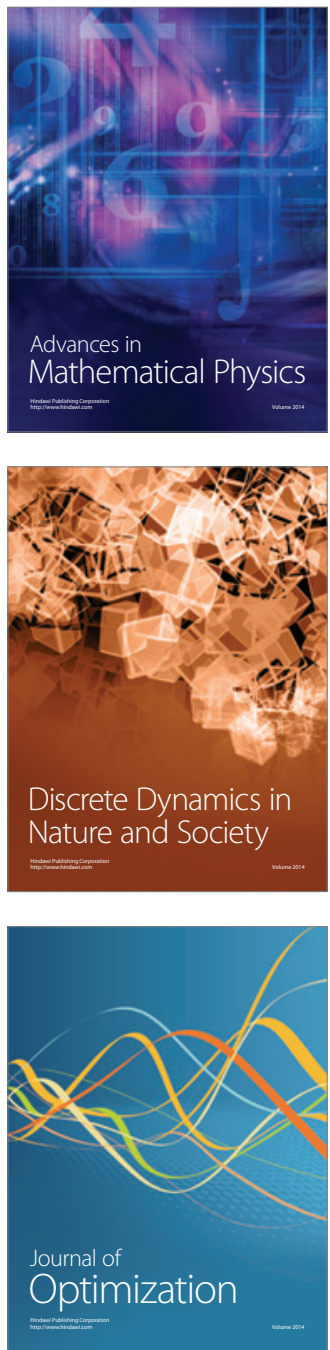